\title{
Electrical cardioversion resulting in ventricular fibrillation
}

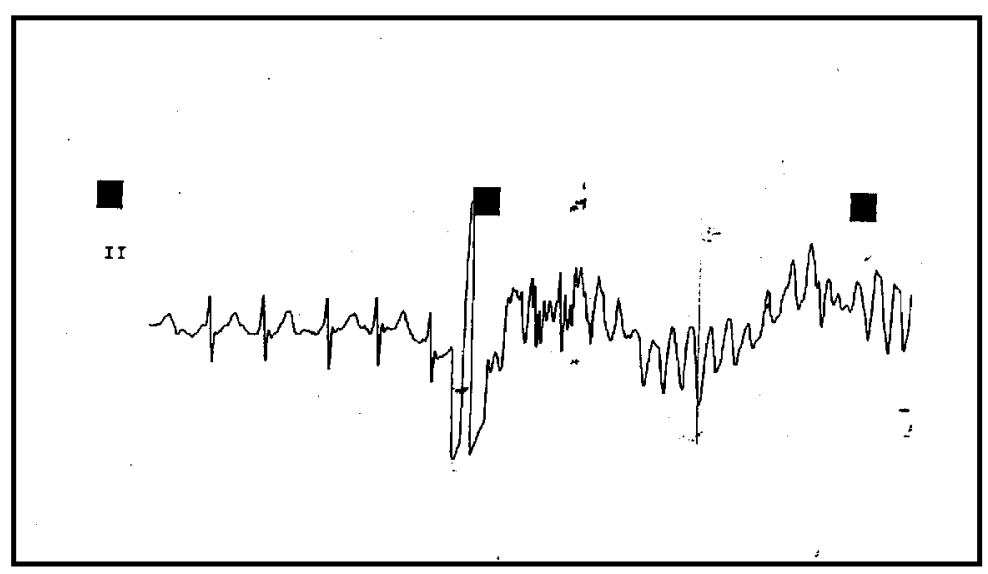

Fig. 1

A 50-year-old man presented to a community hospital with palpitations for approximately two weeks. ECG revealed atrial fibrillation. The patient was treated with warfarin for three weeks and then re-submitted for cardioversion [1]. Cardioversion was performed with a Bruker Defigard 2002 (Bruker, Ettlingen, Germany) with 25 Ws, the procedure was performed according to the recommendations from the handbook. Probably due to synchronization failure ventricular fibrillation occurred (Fig. 1), which was terminated by defibrillation with $100 \mathrm{Ws} 30$ seconds later, resulting in stable sinus rhythm. The patient was discharged at well-being. Synchronization failures due to inappropriate performance by the operator and due to accidental $\mathrm{T}$ wave synchronization have been reported [2]. An alternative explanation for this incident can be found in the very low energy applied in this case in the attempt to terminate atrial fibrillation. An initial energy of 200J or higher is recommended for electrical cardioversion of AF to prevent incomplete depolarisation which could promote ventricular fibrillation [3, 4]. Repetitive analysis of the software by the manufacturer did not reveal a defect in the software of the defibrillator.

Sandra Fortunat, Eva Werba, and Georg Röggla

\section{References}

1. Pritchet ELC (1991) Management of atrial fibrillation. N Engl J M 326: 1264-1271

2. Ebrahimi R, Rubin SA (1994) Electrical cardioversion resulting in death from synchronization failure. Am J Cardiol 74: 100102

3. ACC/AHA/ESC (2001) Guidelines for the management of patients with atrial fibrillation: executive summary. A report of the American College of Cardiology/American Heart Association Task Force on Practice Guidelines and the European Society of Cardiology Committee for Practice Guidelines and Policy Conferences (Committee to Develop Guidelines for the Management of Patients with Atrial Fibrillation). Developed in collaboration with the North American Society of Pacing and Electrophysiology. Circulation 104: 2118-2150

4. Falk RH, Layman TE, Duvernoy WF (1995) Comments on: electrical cardioversion resulting in death from synchronization failure. Am J Cardiol 75: 551-553

Key words: Cardioversion, synchronization failure, ventricular fibrillation.

Correspondence: Prim. Doz. Dr. Georg Röggla, Abteilung für Innere Medizin, A.ö. KH Neunkirchen, Peischingerstraße 19, 2620 Neunkirchen, Austria, E-mail: roeggla.interne@khneunkirchen.at 